\title{
Anticoagulation use and Hemorrhagic Stroke in SARS-CoV-2 Patients Treated at a New York Healthcare System
}

\author{
Alexandra Kvernland ${ }^{1,7^{*+}} \oplus$, Arooshi Kumar ${ }^{1+}$, Shadi Yaghi ${ }^{1}$, Eytan Raz ${ }^{2}$, Jennifer Frontera ${ }^{1,3}$, Ariane Lewis ${ }^{1,3}$, \\ Barry Czeisler, ${ }^{1,3}$, D. Ethan Kahn 1,3, Ting Zhou 1,3, Koto Ishida', Jose Torres' ${ }^{1}$, Howard A. Riina ${ }^{3}$, Maksim Shapiro², \\ Erez Nossek ${ }^{3}$, Peter K. Nelson 2,3, Omar Tanweer ${ }^{3}$, David Gordon ${ }^{3}$, Rajan Jain 2,3, Seena Dehkharghani², \\ Nils Henninger ${ }^{4}$, Adam de Havenon ${ }^{5}$, Brian Mac Grory ${ }^{6}$, Aaron Lord ${ }^{1,3}$ and Kara Melmed ${ }^{1,3}$
}

(C) 2020 Springer Science+Business Media, LLC, part of Springer Nature and Neurocritical Care Society

\begin{abstract}
Background and Purpose: While the thrombotic complications of COVID-19 have been well described, there are limited data on clinically significant bleeding complications including hemorrhagic stroke. The clinical characteristics, underlying stroke mechanism, and outcomes in this particular subset of patients are especially salient as therapeutic anticoagulation becomes increasingly common in the treatment and prevention of thrombotic complications of COVID-19.

Methods: We conducted a retrospective cohort study of patients with hemorrhagic stroke (both non-traumatic intracerebral hemorrhage and spontaneous non-aneurysmal subarachnoid hemorrhage) who were hospitalized between March 1, 2020, and May 15, 2020, within a major healthcare system in New York, during the coronavirus pandemic. Patients with hemorrhagic stroke on admission and who developed hemorrhage during hospitalization were both included. We compared the clinical characteristics of patients with hemorrhagic stroke and COVID-19 to those without COVID-19 admitted to our hospital system between March 1, 2020, and May 15, 2020 (contemporary controls), and March 1, 2019, and May 15, 2019 (historical controls). Demographic variables and clinical characteristics between the individual groups were compared using Fischer's exact test for categorical variables and nonparametric test for continuous variables. We adjusted for multiple comparisons using the Bonferroni method.

Results: During the study period in 2020, out of 4071 patients who were hospitalized with COVID-19, we identified 19 (0.5\%) with hemorrhagic stroke. Of all COVID-19 with hemorrhagic stroke, only three had isolated non-aneurysmal SAH with no associated intraparenchymal hemorrhage. Among hemorrhagic stroke in patients with COVID-19, coagulopathy was the most common etiology (73.7\%); empiric anticoagulation was started in $89.5 \%$ of these patients versus $4.2 \%$ in contemporary controls $(p \leq .001)$ and $10.0 \%$ in historical controls $(p \leq .001)$. Compared to contemporary and historical controls, patients with COVID-19 had higher initial NIHSS scores, INR, PTT, and fibrinogen levels. Patients with COVID-19 also had higher rates of in-hospital mortality ( $84.6 \%$ vs. $4.6 \%, p \leq 0.001)$. Sensitivity analyses excluding patients with strictly subarachnoid hemorrhage yielded similar results.
\end{abstract}

\footnotetext{
*Correspondence: Alexandra.Kvernland@nyulangone.org

${ }^{\dagger}$ Alexandra Kvernland and Arooshi Kumar are co-first authors.

${ }^{7}$ Department of Neurology, New York University Grossman School

of Medicine, 530 First Avenue HCC-5A, New York, NY 10016, USA

Full list of author information is available at the end of the article
}

\section{实 Springer}


Conclusion: We observed an overall low rate of imaging-confirmed hemorrhagic stroke among patients hospitalized with COVID-19. Most hemorrhages in patients with COVID-19 infection occurred in the setting of therapeutic anticoagulation and were associated with increased mortality. Further studies are needed to evaluate the safety and efficacy of therapeutic anticoagulation in patients with COVID-19.

Keywords: Coronavirus, COVID-19, Pandemic, Hemorrhagic stroke, Intracerebral hemorrhage, Subarachnoid hemorrhage, Coagulopathy

\section{Introduction}

Coronavirus disease 2019 (COVID-19) is an international pandemic, caused by the severe acute respiratory syndrome CoV-2 coronavirus (SARS-CoV-2). As of May 22, 2020, over 1.5 million people in the USA have been infected with the virus, and over 95,000 have died as per the Center for Disease Control [1]. In New York State, over 350,000 people have contracted the disease and more than 29,000 have died [1]. Based on these statistics, New York State currently accounts for approximately $30 \%$ of all COVID-19-related deaths reported thus far in the USA.

While there have been some observational studies identifying cerebrovascular disease as a clinical manifestation of COVID-19, hemorrhagic stroke has not been well studied in this population [2-8]. A recent study characterized 33 COVID-19-positive patients with hemorrhagic stroke at our institution as parenchymal with mass effect and herniation (15.2\%), punctate (25\%), and small- to moderate-sized (60.7\%) and large hemorrhages without evidence of herniation (14.3\%), but this study included patients with hemorrhagic transformation of ischemic infarcts and did not include a comparator group [2].

Identifying factors associated with hemorrhagic stroke in patients with COVID-19 can facilitate understanding of the mechanisms contributing to hemorrhagic stroke and assist in bleed prevention, diagnosis, and management. As many intensive care units are developing empiric anticoagulation protocols to address the hypercoagulable state associated with COVID-19, understanding the mechanisms predisposing patients to bleeding is imperative. Moreover, there are limited data on the incidence of hemorrhagic stroke patients with COVID-19.

In this study, we aim to characterize hemorrhagic stroke in patients with COVID-19 from a large healthcare system with a diverse patient population in the New York metropolitan area and compare these parameters to those of both contemporary and historical controls without COVID-19. We expand upon previous studies from our institution [2] over a larger time period with the addition of the comparator groups.

\section{Methods}

\section{Study Population}

Institutional review board approval was obtained from NYU Langone Health, and informed consent was waived. Data from the study are available for sharing upon reasonable request to the corresponding author. This is a retrospective observational study including all consecutive patients with radiologic confirmation of acute hemorrhagic stroke during the specified time frame patients admitted to one of three comprehensive stroke centers in New York: NYU Langone Manhattan, NYU Langone Brooklyn (Sunset Park, Brooklyn), and NYU Langone Winthrop (Mineola, Long Island) with hemorrhagic stroke (intracerebral hemorrhage (ICH) or non-aneurysmal subarachnoid hemorrhage (SAH)) hospitalized between March 1, 2020, and May 15, 2020. Patients with hemorrhagic stroke on admission and who developed hemorrhage during hospitalization were both included. Patients with positive reverse-transcriptase polymerase chain reaction (RT-PCR) SARS-CoV-2 test were included in the study group. Patients with negative RT-PCR SAR$\mathrm{SCoV}-2$ were included in the contemporary control group. We included consecutive patients from NYU Langone Manhattan and NYU Langone Brooklyn who were discharged with a diagnosis of non-traumatic acute hemorrhagic stroke (ICH and SAH) between March 1, 2019, and May 15, 2019, as a historical control group. Patients with confirmed hemorrhagic transformation of ischemic stroke, traumatic intracranial hemorrhage, or aneurysmal $\mathrm{SAH}$ were excluded from this study.

\section{Stroke Assessment and Classification}

Patients underwent a standard diagnostic evaluation including brain imaging and intracranial vascular imaging for clinical purposes. Hemorrhagic stroke was classified as either ICH or non-aneurysmal SAH. Bleed etiology was classified as secondary to hypertension, brain metastases, coagulopathy, vascular abnormality (arteriovenous malformation), other (including cerebral amyloid angiopathy), or unknown. ICH location was defined as primarily deep, primarily cortical, deep and cortical, or multifocal. Size of hemorrhage was calculated by two independent adjudicators using the $\mathrm{ABC} / 2$ 
method [9]. ICH score was calculated for primary intracerebral hemorrhages [10].

\section{Study Variables}

We abstracted demographic, clinical, laboratory, and imaging variables, in-hospital events and treatments, and discharge outcomes from the medical records of all study patients.

Demographic variables included age, sex, and race/ethnicity. Clinical variables included history of hypertension, diabetes mellitus, hyperlipidemia, atrial fibrillation, prior intracerebral hemorrhage, home medications (including antiplatelets and anticoagulants), presenting symptoms, initial National Institutes of Health Stroke Scale (NIHSS) score, date of likely stroke symptom onset (based on last known well or abstractors' clinical judgment), ICH score, and the systolic blood pressure (closest to the time of hemorrhagic stroke diagnosis). Laboratory variables were obtained closest to the time of hemorrhagic stroke diagnosis and included platelet count, D-dimer level, C-reactive protein level, international normalized ratio (INR), prothrombin time (PT), activated partial thromboplastin time (aPTT), and fibrinogen. Neutrophil-to-lymphocyte ratio (NLR) was calculated by dividing absolute neutrophil count by absolute lymphocyte count on the complete blood count differential. Imaging variables included location of hemorrhage (deep, cortical, deep and cortical, or multifocal), volume of ICH (in cubic centimeters), and presence or absence of intraventricular hemorrhage. Inhospital events and treatments included use of anticoagulant therapy, mechanical ventilation, interval between initiation of anticoagulation or antiplatelet therapy and onset of hemorrhagic stroke (in days), and seizure. Discharge outcome was in-hospital mortality.

\section{COVID-19 Screening and Diagnosis}

Evaluation for all patients at first provider contact included history of recent COVID-19 exposure, history of symptoms associated with COVID-19, and a chest $\mathrm{x}$-ray. Patients with a positive screen underwent formal testing using a RT-PCR SARS-CoV-2 test with a nasopharyngeal swab. This test established COVID-19 status as either positive or negative.

\section{Statistical analysis}

We divided patients into three groups: Group A included patients with hemorrhagic stroke and COVID-19 (cases), group B included contemporary patients with hemorrhagic stroke without COVID-19 (contemporary controls), and group $\mathrm{C}$ included historical patients with hemorrhagic stroke without COVID-19 over the same time frame (historical controls). We compared demographic variables and clinical characteristics between the individual groups using Fischer's exact test for categorical variables and nonparametric test for continuous variables. We adjusted for multiple comparisons using the Bonferonni method. Analysis was performed using SPSS version 25.0 (Chicago, IL), and a two-sided $p<0.05$ was considered significant.

\section{Results \\ Characteristics of Patients with COVID-19 and Hemorrhagic Stroke}

Among a total of 4071 patients who were hospitalized with confirmed COVID-19 during the study period, we identified 34 patients with hemorrhagic stroke; 15 of these were excluded due to a definitive diagnosis of hemorrhagic transformation of ischemic stroke and 19 $(0.5 \%)$ were included (Fig. 1). A clinical summary of all COVID-19 patients with hemorrhagic stroke is included in Table 1. The median age of patients with COVID19-associated hemorrhagic stroke was 60 years; $78.9 \%$ were men. Prior to diagnosis of hemorrhagic stroke, these patients were empirically started on the following treatments: hydroxychloroquine $(73.6 \% ; 14 / 19)$, interleukin-6 inhibitor (26.3\%; 5/19), and lopinavir/ritonavir (5.26\%; 1/19). Patients with COVID-19 and hemorrhagic stroke were critically ill; $84.2 \%$ required mechanical ventilation; and the mortality rate at hospital discharge was $84.6 \%$ and, at the time of last follow-up, was $57.9 \%$. Of all COVID-19 with hemorrhagic stroke, only three had isolated non-aneurysmal SAH with no associated intraparenchymal hemorrhage. Coagulopathy was the most common etiology of hemorrhagic stroke in patients with COVID-19 at $73.7 \%$. Two patients were not on any anticoagulation prior to hemorrhage. Of the 17 COVID-19 patients on anticoagulation, the goal intensity was full therapeutic range. While on anticoagulation therapy and within 5 days prior to hemorrhagic stroke diagnosis $58.8 \%(10 / 17)$ were supratherapeutic, $29.4 \%(5 / 17)$ were consistently in therapeutic range, and $11.8 \%(2 / 17)$ were at some point therapeutic range but subtherapeutic just prior to diagnosis. The indication for anticoagulation was suspected COVID-19 hypercoagulability based on our internal protocol in 64.7\% (11/17) patients. All other indications are summarized in Table 1 . No patients across any cohort had history of SAH, and only one patient in the historical control group had history of ischemic stroke.

\section{Univariate Analyses}

The characteristics of patients with COVID-19 hemorrhagic stroke as compared to contemporary and history controls are summarized in Table 2. Compared to contemporary controls, patients with COVID-19 and hemorrhagic stroke had higher initial NIHSS scores [median 

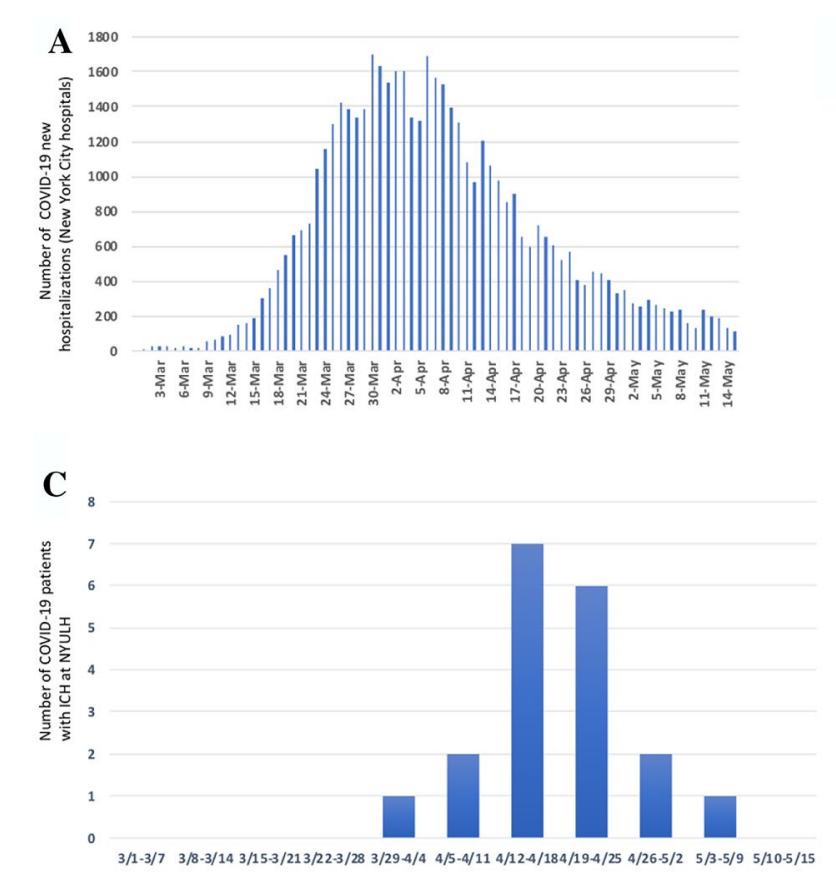

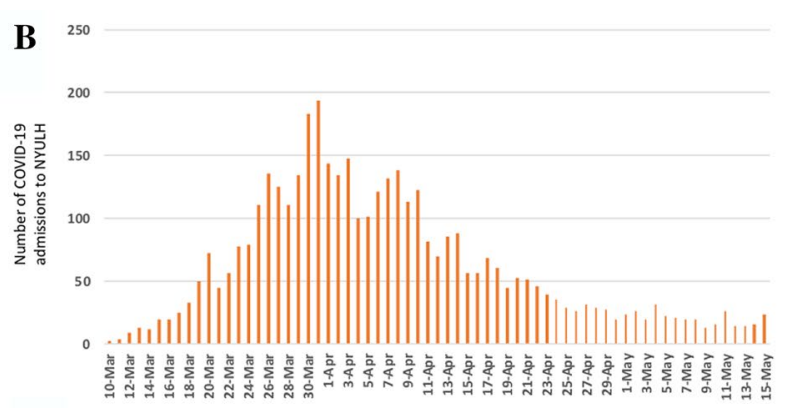

D

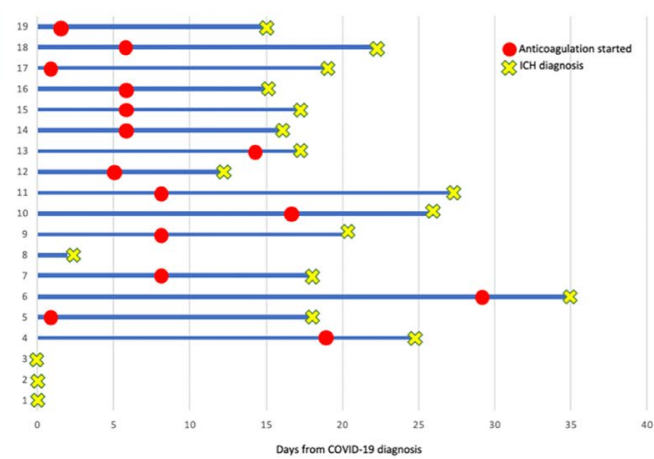

Fig. 1 The number of COVID-19 hospitalizations and hemorrhagic strokes during the study period. a Overall number of COVID-19 hospitalizations in all of NYC hospitalizations. b The number of COVID-19 hospitalizations at NYU Langone Health System over the study period. c Number of COVID-19 patients diagnosed with hemorrhagic stroke. $\mathbf{d}$ Time graph of point at which anticoagulation was started and when hemorrhagic stroke was diagnosed

(IQR) NIHSS score: 28 (23-30) vs. 13 (3-20), $p \leq 0.001)$ ], were less likely to have strictly deep hemorrhage $[6.25 \%$ vs. $56.0 \%, p=0.002]$, and had lower systolic blood pressure (SBP) closest to time of ICH diagnosis [median (IQR) SBP: 133 (114-136) vs. 158 (140-176), $p=0.001$ ]. These patients were more likely to have been on anticoagulation prior to diagnosis of hemorrhagic stroke [89.5\% vs $4.2 \%, p=0.001$ ] (identified in Fig. $1 \mathrm{D}$ and depicted in Fig. 2) and had higher INR levels [median (IQR): 1.3 $(1.2-2.7)$ vs. $1.1(1.0-1.1), p \leq 0.001]$, higher PTT levels [median (IQR): 64.9 (37.7-94.6) vs. 31.7 (28.7-37.1), $p=0.001$ ], and higher fibrinogen levels [median (IQR): 669 (578-785) vs. 336 (249-422), $p=0.004]$ closest to time of ICH diagnosis. There was a higher rate of inhospital mortality for patients with COVID-19 [84.6\% vs. $4.6 \%, p \leq 0.001$ ]. Hypertension was the most common etiology for contemporary controls (45.8\%).

Compared to historical controls, patients with COVID19 and hemorrhagic stroke had higher initial NIHSS scores [median (IQR) NIHSS score: $28(23-30)$ vs. 6 $(1-13), p \leq 0.001)]$ and higher ICH scores [median (IQR): $2(1-4)$ vs. $2(1-3), p=0.015)]$. They were less likely to have history of hypertension [ $42.1 \%$ vs. $80.0 \%, p=0.022$ ], were less likely to have strictly deep ICH $[6.25 \%$ vs. $56.5 \%, p=0.002]$, and had lower SBP closest to time of
ICH diagnosis [median (IQR) SBP: 133 (114-136) vs. 157 (131-180), $p=0.006]$. Patients with COVID-19 and hemorrhagic stroke were more likely to have been on anticoagulation prior to diagnosis of hemorrhagic stroke [89.5\% vs $10.0 \%, p \leq 0.001$ ] and had higher INR levels [median (IQR): $1.3(1.2-2.70)$ vs. 1.1 (1.0-1.2), $p \leq 0.001]$, PTT levels [median (IQR): 64.9 (37.7-94.6) vs. 31.9 (29.8$34.2), p \leq 0.001$ ], and fibrinogen levels [median (IQR): 669 (578-785) vs. 319 (292-397), $p=0.004$ ] closest to the time of hemorrhagic stroke diagnosis. Patients with COVID-19 and hemorrhagic stroke had a higher rate of in-hospital mortality than historical controls $[84.6 \%$ vs. $5.0 \%, p \leq 0.001]$. Hypertension was the most common etiology for historical controls (35.0\%). Though patients with COVID-19 had higher median NLR ratios, there was no significant difference in NLR ratios when compared to either control group.

\section{Sensitivity Analyses}

In sensitivity analyses excluding strictly SAH patients when compared to contemporary controls, patients with COVID-19 and hemorrhagic stroke were more likely to be on anticoagulation therapy $(90.9 \%$ vs. $5.0 \%$, $p<0.001$ ), were less likely to have strictly deep intraparenchymal hemorrhage $(9.1 \%$ vs. $65.0 \%, p=0.004)$, 


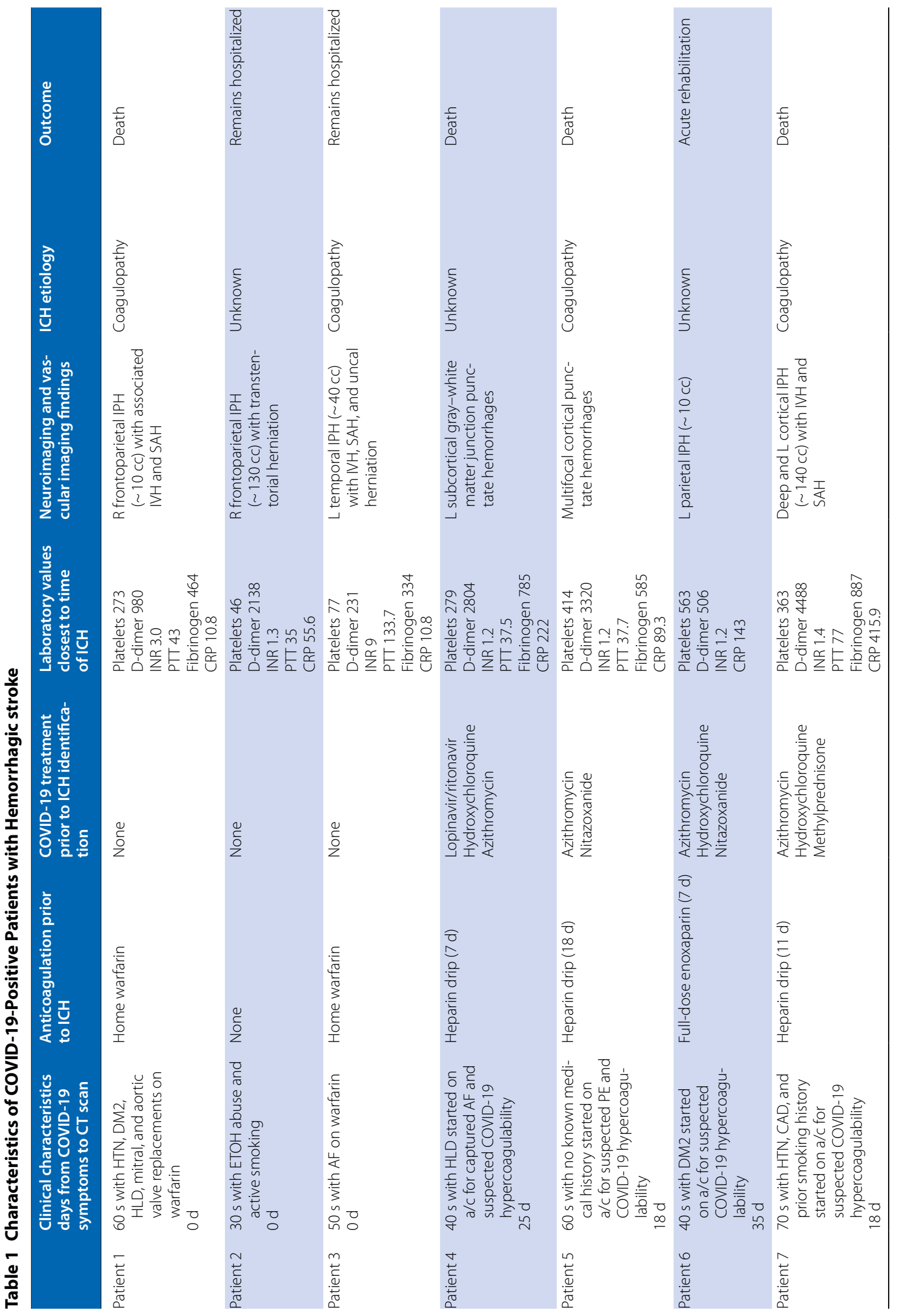




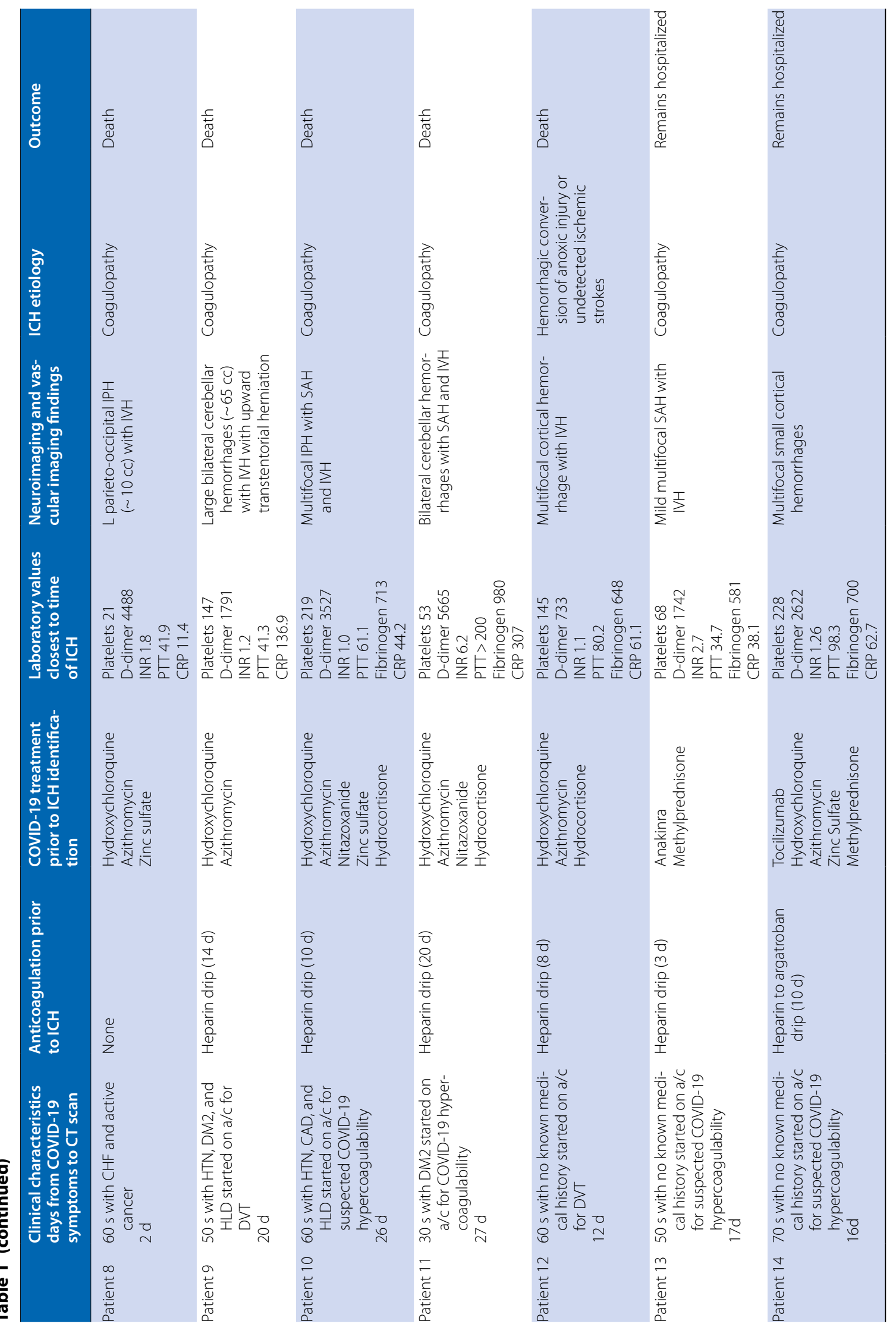




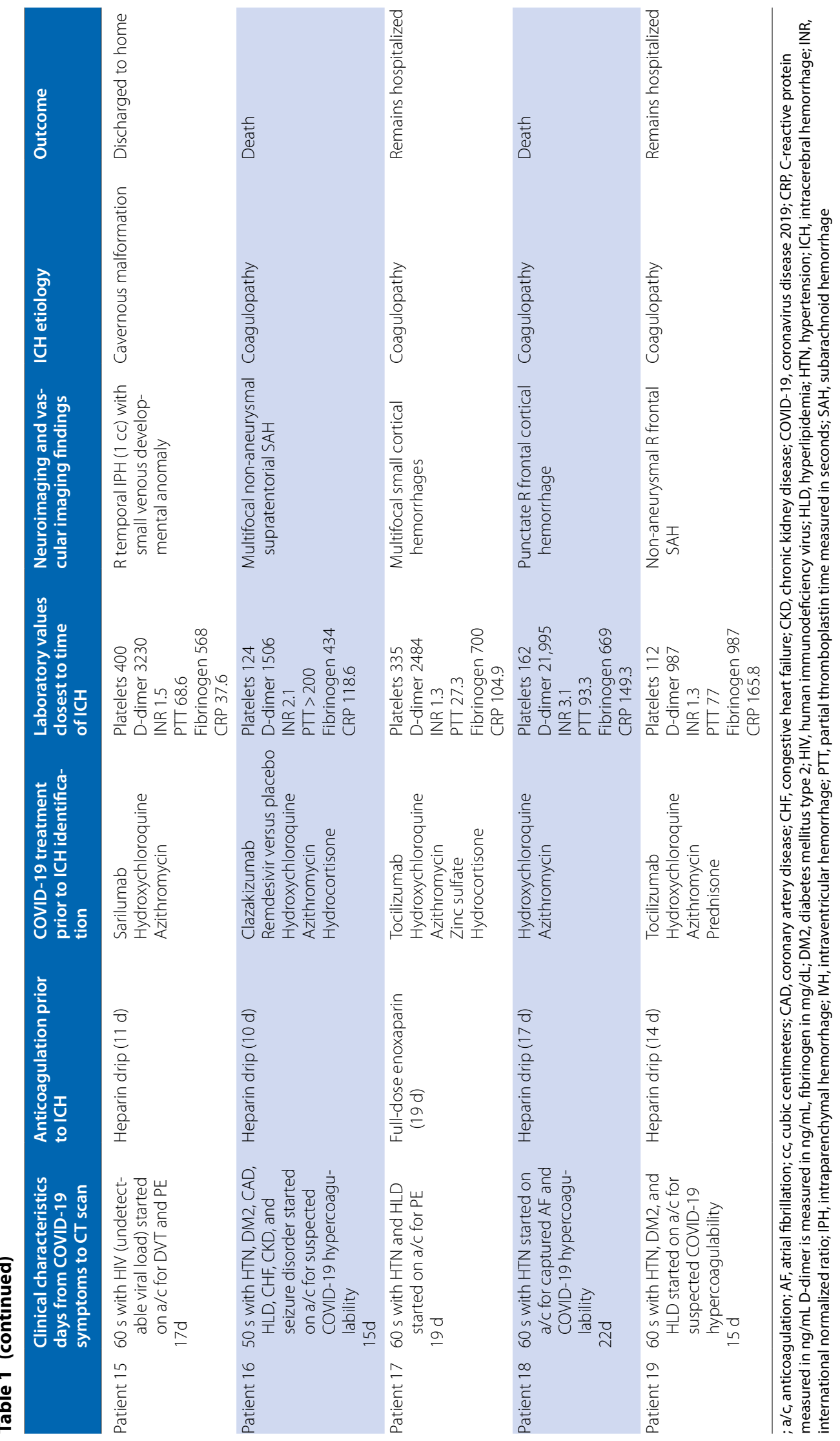


Table 2 Demographic and Clinical Characteristics of Patients with Hemorrhagic Stroke and COVID-19 versus Contemporary and Historical Controls without COVID-19 infection

\begin{tabular}{|c|c|c|c|c|c|}
\hline & $\begin{array}{l}\text { COVID-19 posi- } \\
\text { tive }(A)(n=19)\end{array}$ & $\begin{array}{l}\text { Contemporary } \\
\text { controls (B) } \\
(n=24)\end{array}$ & $\begin{array}{l}\text { Historical } \\
\text { controls (C) } \\
(n=20)\end{array}$ & $p$ value ( $A$ vs. $B$ ) & $p$ value ( $A$ vs. $C$ ) \\
\hline Age (median, IQR) & $60(51-63)$ & $68(49-74)$ & $59(50-81)$ & 0.073 & 0.863 \\
\hline Male (\%) & $78.9 \%(15)$ & $66.7 \%(16)$ & $70.0 \%(14)$ & 0.500 & 0.716 \\
\hline Hypertension (\%) & $42.1 \%(8)$ & $70.8 \%(17)$ & $80.0 \%(16)$ & 0.071 & 0.022 \\
\hline Diabetes Type 2(\%) & $31.6 \%(6)$ & $29.2 \%(7)$ & $25.0 \%(5)$ & 1.000 & 0.731 \\
\hline Hyperlipidemia (\%) & $36.8 \%(7)$ & $25.0 \%(6)$ & $25.0 \%(5)$ & 0.509 & 0.501 \\
\hline Atrial fibrillation (\%) & $5.26 \%(1)$ & $12.5 \%(3)$ & $0 \%(0)$ & 0.618 & .487 \\
\hline Strictly deep intracerebral hemorrhage location & $6.25 \%(1 / 16)$ & $56.5 \%(13 / 23)$ & $62.5 \%(10 / 16)$ & 0.002 & 0.002 \\
\hline Prior intracerebral hemorrhage (\%) & $0 \%(0)$ & $16.7 \%(4)$ & $10.0 \%(2)$ & 0.118 & 0.487 \\
\hline Antiplatelet therapy at home (\%) & $21.1 \%(4)$ & $33.3 \%(8)$ & $30.0 \%(6)$ & 0.500 & 0.716 \\
\hline Anticoagulation at home (\%) & $10.5 \%(2)$ & $4.2 \%(1)$ & $10.0 \%(2)$ & 0.575 & 1.000 \\
\hline Initial NIHSS score (median, IQR) & $28(23-30)$ & $13(3-20)$ & $6(1-13)$ & $<0.001$ & $<0.001$ \\
\hline ICH score (median, IQR) & $2(1-4)$ & $2(1-3)$ & $1(0-1)$ & 0.912 & 0.015 \\
\hline $\begin{array}{l}\text { Anticoagulation prior to hemorrhagic stroke diagnosis } \\
(\%)\end{array}$ & $89.5(17)$ & $4.2 \%(1)$ & $10.0 \%(2)$ & $<0.001$ & $<0.001$ \\
\hline Systolic blood pressure (median, IQR) & $133(114-136)$ & $158(140-176)$ & $157(131-180)$ & $<0.001$ & 0.006 \\
\hline Platelet count (median, IQR) & $162(77-335)$ & $238(189-263)$ & $251(188-301)$ & 0.632 & 0.415 \\
\hline INR (median, IQR) & $1.3(1.2-2.7)$ & $1.1(1.0-1.1)$ & $1.1(1.0-1.2)$ & $<0.001$ & $<0.001$ \\
\hline PTT (median, IQR) & $64.9(37.7-94.6)$ & $31.7(28.7-37.1)$ & $31.9(29.8-34.2)$ & 0.001 & $<0.001$ \\
\hline Fibrinogen (median, IQR) & $669(578-785)$ & $336(249-422)$ & $319(292-397)$ & 0.004 & 0.004 \\
\hline D-dimer level (median, IQR) & $2484(987-3320)$ & $800(307-3401)$ & N/A & 0.160 & N/A \\
\hline CRP level (median, IQR) & $89.3(38.1-149.3)$ & $41.2(3.3-132.8)$ & N/A & 0.245 & N/A \\
\hline Neutrophil-to-lymphocyte ratio (NLR) & $9.82(5.39-15.30)$ & $6.73(2.43-10.91)$ & $5.84(2.31-11.77)$ & 0.215 & 0.150 \\
\hline $\mathrm{ICH}$ volume (median cm, IQR) & $37.0(9.1-98.7)$ & $11.0(2.9-32.1)$ & $5.0(1.9-43.5)$ & 0.763 & 0.462 \\
\hline Intraventricular hemorrhage (\%) & $36.8 \%(7)$ & $58.3 \%(14)$ & $40.0 \%(8)$ & 0.223 & 1.000 \\
\hline ICH etiology (\%) & & & & $<0.001$ & $<0.001$ \\
\hline Hypertension & $0 \%(0)$ & $45.8 \%(11)$ & $35.0 \%(7)$ & & \\
\hline Brain tumor & $0 \%(0)$ & $12.5 \%(3)$ & $0 \%(0)$ & & \\
\hline Vascular malformation & $5.26 \%(1)$ & $8.3 \%(2)$ & $15.0 \%(3)$ & & \\
\hline Coagulopathy & $73.7 \%(14)$ & $0 \%(0)$ & $5.0 \%(1)$ & & \\
\hline Other & $5.26 \%(1)$ & $8.3 \%(2)$ & $25.0 \%(5)$ & & \\
\hline Unknown & $15.8 \%(3)$ & $25.0 \%(6)$ & $20.0 \%(4)$ & & \\
\hline In-hospital death (\%) & $84.6 \%(11 / 13)$ & $4.6 \%(1 / 22)$ & $5.0 \%(1 / 20)$ & $<0.001$ & $<0.001$ \\
\hline
\end{tabular}

COVID-19, coronavirus disease 2019; CRP, C-reactive protein measured in $\mathrm{ng} / \mathrm{mL}$; D-dimer is measured in ng/mL, fibrinogen in $\mathrm{mg} / \mathrm{dL}$; ICH, intracerebral hemorrhage; INR, international normalized ratio; IQR, interquartile range; NIHSS, National Institutes of Health Stroke Scale; PTT, partial thromboplastin time measured in seconds; SBP, systolic blood pressure

and had higher mortality at discharge $(77.8 \%$ vs. $5.6 \%$, $p<0.001)$. Furthermore, when compared to historical controls, patients with COVID-19 and hemorrhagic stroke were more likely to be on anticoagulation therapy $(90.9 \%$ vs. $11.8 \%, p<0.001)$, were less likely to have strictly deep intraparenchymal hemorrhage $(9.1 \%$ vs. $71.4 \%, p=0.004)$, and had higher mortality at discharge $(77.8 \%$ vs. $5.9 \%, \mathrm{p}<0.001)$.

\section{Discussion}

\section{Main Findings}

Our study demonstrates that the risk of hemorrhagic stroke in patients hospitalized with COVID-19 infection is low. Coagulopathy in the setting of therapeutic anticoagulation use, as evidenced by elevated PTT and INR levels, may be a driving factor in patients with hemorrhagic stroke in the setting of COVID-19 

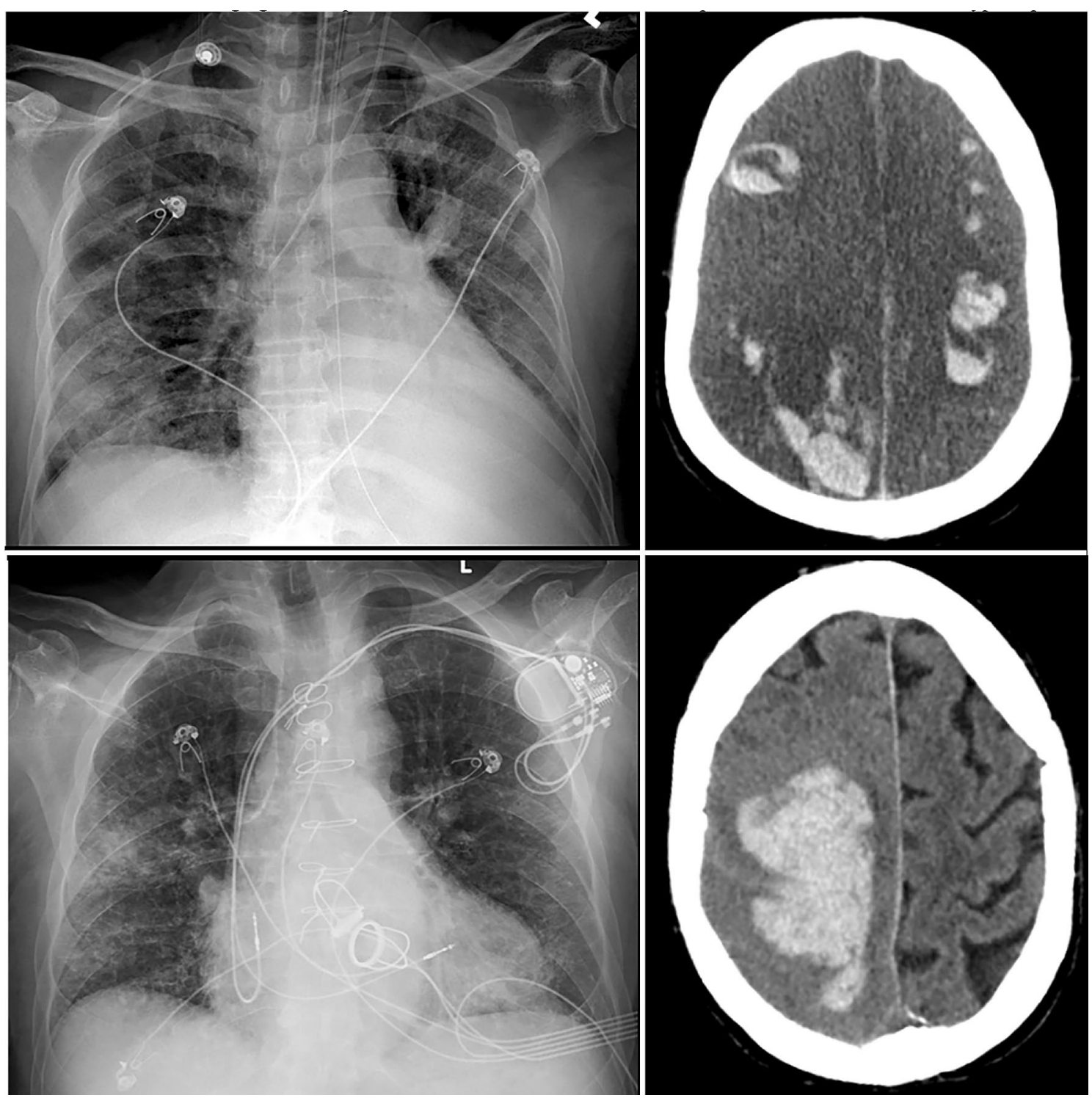

Fig. 2 Two patients with hemorrhagic stroke in the setting of coronavirus disease 2019 (COVID-19). The left column shows thoracic X-ray, and the right column shows brain non-contrast CT for both patients. CT demonstrates primarily peripheral, parenchymal hematomas in patient 1 (top row), including a large, dominant paramedian parietal hemorrhage on the right and generalized sulcal effacement and cerebral edema. Patient 2 (bottom row) demonstrates a solitary but large parenchymal hematoma in the perirolandic vertex of the right hemisphere with severe, unilateral sulcal effacement. Thoracic imaging in both patients revealed severe, multifocal airspace disease consistent with atypical pneumonia

(89.5\%) when compared to contemporary controls (4.2\%) and historical controls (10.0\%). Strictly deep intracerebral hemorrhages were less common in patients with COVID-19 when compared to contemporary and historical controls, in line with the lower rate of hypertension and the lower systolic blood pressure at the time the hemorrhage was diagnosed.

\section{Mechanisms of Hemorrhage}

There are multiple possible mechanisms contributing to hemorrhagic stroke in patients with COVID-19. COVID19 has been associated with modest decreases in platelet count, and prolonged prothrombin time. [11]. In addition, evidence suggests the overactivation of the fibrinolytic cascade in patients with COVID-19 [11]. In fact, 
patients with severe acute respiratory syndrome coronavirus (SARS-CoV-1) were reported to have increased tissue plasminogen activator and thrombomodulin plasma levels [12], both of which are associated with increased propensity for hemorrhagic complications. Therefore, it is possible that a coagulopathy in the setting of activation of intrinsic and extrinsic fibrinolytic processes predisposed these patients to hemorrhagic stroke.

Most importantly, the majority of patients with COVID-19 (89.5\%) in this study were started on fulldose anticoagulation per our internal protocol due to elevated D-dimer levels (most commonly unfractionated heparin) for prevention of thrombotic complications associated with COVID-19 that are increasingly being reported [11, 13, 14]. Multiple societal guidelines, which were developed after our study period, specifically from the American Society of Hematology and American Society of CHEST Physicians do not recommend full-dose anticoagulation for prevention of thromboembolism in COVID-19 given the unclear benefits and potential high risk of bleeding in this vulnerable population [15, 16]. Of note, majority of the COVD-19 patients in our study cohort were found to be supratherapeutic while on anticoagulation prior to hemorrhagic stroke diagnosis, suggesting inadvertent outcomes of being on full-dose therapy. Moreover, the benefit of preventing thrombosis in these patients cannot be determined in this study.

In addition, it is possible that SARS-CoV-2 has both direct and indirect effects on the cerebrovascular system that increases the risk of hemorrhage through various potential mechanisms $[3,17,18]$. The presence of SARS$\mathrm{CoV}-1$ virus has been demonstrated in human brains, and there are multiple cases of central nervous system involvement of other similar coronaviruses, highlighting its neuro-invasive potential [17]. SARS-CoV-2 binds to ACE2 receptors, which are expressed by neurons and glial cells, raising the possibility of direct viral neurologic injury [15]. Additionally, ACE2 receptors are highly concentrated on the endothelial cells of blood vessels, and when attacked by SARS-CoV-2, may result in disruption of the blood brain barrier and abnormal cerebral autoregulation [19]. A recent case series demonstrated significant vascular angiogenesis in the lungs of patients with COVID-19, underscoring the need for additional autopsy data to validate similar changes in cerebral vasculature [20].

Another proposed mechanism involves indirect injury from massive cytokine release which can result in breakdown of the blood brain barrier, increasing the risk of hemorrhagic stroke [18]. Interestingly, the median NLR trended higher for COVID-19 patients as compared to contemporary and historical controls although not significantly different, possibly due to the size of our study.
Further, it has been suggested that pulmonary edema seen in early phases of COVID-19 infection is due to activation of bradykinin receptors on vascular endothelial cells, a pathway that is dysregulated in the setting of ACE2 deficiency; there may be similar vascular leakage occurring in small blood vessels of the brain [21]. An important consideration is that while we excluded hemorrhages that were clearly the result of hemorrhagic transformation of ischemic stroke, it remains possible that some of the hemorrhages in our cohort are the result of hemorrhagic conversion of primary ischemic strokes or hypoxic injury, especially as early anticoagulation [22, 23] and bridging therapy [24] with low molecular weight heparin is associated with an increased risk of hemorrhagic transformation.

\section{Potential Therapeutic Implications}

Our data have several salient implications for clinical care. First, rapid neurologic assessment of patients with COVID-19 must be prioritized as many hemorrhagic strokes were incidentally identified. Attempting to wean or stop sedation for interval neurologic examinations, especially if there is clinical suspicion for acute stroke, and particularly for patients on therapeutic anticoagulation is imperative. In patients who are intubated and sedated, it is reasonable to obtain a screening CT prior to initiation of therapeutic anticoagulation, especially to evaluate for evidence hypoxic ischemic injury. In patients with abnormal elevation in coagulation laboratories, a lower threshold for obtaining a screening CT may be considered, especially when the neurologic examination is confounded by sedation.

COVID-19 patients are at risk of thrombotic events and often require either prophylactic or therapeutic anticoagulation at some point during their hospitalization. In this setting, clinicians must weigh the risk of hemorrhagic stroke when deciding on the agent and timing of anticoagulation initiation. Additional randomized controlled studies are needed, such as the ongoing PROTECT COVID clinical trial, to support the use of therapeutic anticoagulation for primary prevention of thrombotic complications in patients with COVID-19. Moreover, it is important that anticoagulation protocols account for the risk of hemorrhagic stroke given its association with anticoagulation use in our study and the increased mortality of hemorrhagic stroke which may have been partially related to the occurrence of hemorrhagic stroke in our study.

\section{Strengths and Limitations}

There are a number of limitations inherent to our study. First, our sample size is small, retrospective, and observational, with the potential for selection 
bias. Importantly, in order to capture all hemorrhagic strokes in the time period specified across our hospital system, we included both ICH and non-aneurysmal SAH patients, two distinct disease processes with very different clinical courses and mortality risks which introduces additional bias. This decision was made as non-aneurysmal SAH can often be attributed to a coagulopathy, and we felt it was important to include these patients in our analysis. It is important to note in sensitivity analysis eliminating purely SAH patients, the mortality was still significantly higher than prior controls. Though all efforts were made to include relevant laboratory and diagnostic data, we do not have complete data for all study patients and thus may have missing alternate etiologies of hemorrhagic stroke. However, given several patients were diagnosed with hemorrhagic stroke incidentally rather than based on a change in neurologic status, the time at which hemorrhagic stroke occurred and how it relates to the study variables, captured at time closest to diagnosis, involves some degree of error. Our study may have missed hemorrhagic stroke diagnosis in COVID-19 who faced several barriers to diagnostic stroke workup due to multiple factors including hemodynamic instability, impaired consciousness, or withdrawal of lifesustaining therapies. Many patients were sedated for treatment of COVID-19, and since neurologic examinations occurred sparsely, the probability of missing neurologic changes increases. Moreover, given we did not obtain screening intracranial imaging in all COVID-19 patients, it is possible that many patients expired with undiagnosed hemorrhagic stroke. Additionally, we used the $\mathrm{ABC} / 2$ method to measure hematoma volume. While some studies support its use, there is some evidence suggesting this method is not as accurate as volumetric or planimetric measurement methods [25, 26]. The FDA revoked emergency use of Hydroxychloroquine, after our study period. As such, larger studies are needed to determine how these medications affect mortality. Finally, while our current internal protocol recommends dose adjustment of unfractionated heparin based on anti-Xa levels, we collected PTT as a better validated marker of coagulopathy in our historical sample.

Our study on the other hand has several strengths. While the low number of patients with hemorrhagic stroke limits the generalizability of this study, our cohort of 4071 COVD-19 patients is one of the largest published. It reports several clinical characteristics of a diverse patient population with hemorrhagic stroke in the setting of COVID-19 and, to our knowledge, is the first study to compare these factors to COVID-19-negative contemporary and historical controls.

\section{Conclusion}

We observed a relatively low rate of hemorrhagic stroke in hospitalized patients with COVID-19. The majority of hemorrhagic strokes in patients with COVID19 were thought to be secondary to coagulopathy, as $89.5 \%$ of these patients were on therapeutic dose anticoagulation, a significantly higher percentage than contemporary or historical controls without COVID19. Hemorrhagic stroke in the setting of COVID-19 was associated with an increase in hospital mortality. Larger studies are needed to further validate the clinical characteristics we found significant and further studies to evaluate the risks and benefits of therapeutic anticoagulation in stroke patients with COVID-19.

\section{Author details \\ ${ }^{1}$ Department of Neurology, NYU Langone Medical Center, New York, NY 10016, USA. ${ }^{2}$ Department of Radiology, NYU Langone Medical Center, New York, NY 10016, USA. ${ }^{3}$ Department of Neurosurgery, NYU Langone Medical Center, New York, NY 10016, USA. ${ }^{4}$ Department of Neurology, University of Massachusetts Medical School, Worcester, MA 01655, USA. ${ }^{5}$ Department of Neurology, University of Utah School of Medicine, Salt Lake City, UT 84132 USA. ${ }^{6}$ Department of Neurology, Warren Alpert Medical School of Brown University, Providence, RI 02903, USA. 7 Department of Neurology, New York University Grossman School of Medicine, 530 First Avenue HCC-5A, New York, NY 10016, USA.}

Author Contributions

SY, KM, A Kvernland, and A Kumar designed the study and drafted the manuscript. A Kvernland and A Kumar were involved in data abstraction. SY analyzed the data. All contributing authors revised the manuscript.

\section{Source of Support}

Dr. Yaghi's previous institution received funding from Medtronic for his role in outcome adjudication for Stroke AF; in addition, Dr. Yaghi received funding from Medlink and Massachusetts General Hospital. Dr. Dehkharghani receives research and travel support from iSchemaView. Dr. Henninger is supported by K08NS091499 from the National Institute of Neurological Disorders and Stroke of the National Institutes of Health. All other authors report no relevant disclosures.

\section{Conflict of interest}

The authors declare that they have no conflict of interest.

\section{Ethical Approval}

IRB approval was obtained from NYU Langone Health.

\section{Publisher's Note}

Springer Nature remains neutral with regard to jurisdictional claims in published maps and institutional affiliations.

Received: 9 June 2020 Accepted: 30 July 2020

Published online: 24 August 2020

\section{References}

1. Centers for Disease Control and Prevention. COVID-19 Cases in the U.S. Centers for Disease Control and Prevention. https://www.cdc.gov/ coronavirus/2019-ncov/cases-updates/cases-in-us.html. Published May 22, 2020. Accessed 26 May 2020.

2. Dogra SJR, Cao M, et al. Hemorrhagic stroke and anticoagulation in covid19. J Stroke Cerebrovasc Dis. 2020;29:104984 
3. Mao L, Jin H, Wang M, Hu Y, Chen S, He Q, et al. Neurologic manifestations of hospitalized patients with coronavirus disease 2019 in Wuhan, china. JAMA Neurol. 2020;77:683.

4. Oxley TJ, Mocco J, Majidi S, Kellner CP, Shoirah H, Singh IP, et al. Largevessel stroke as a presenting feature of covid-19 in the young. N Engl J Med. 2020;382:e60.

5. Yaghi S, Ishida K, Torres J, Mac Grory B, Raz E, Humbert K, et al. Sars2-cov-2 and stroke in a New York healthcare system. Stroke. 2020;51:2002.

6. Valderrama EV, Humbert K, Lord A, Frontera J, Yaghi S. Severe acute respiratory syndrome coronavirus 2 infection and ischemic stroke. Stroke. 2020. https://doi.org/10.1161/STROKEAHA.120.030153.

7. Merkler AE, Parikh NS, Mir S, Gupta A, Kamel H, Lin E, et al. Risk of ischemic stroke in patients with coronavirus disease 2019 (covid-19) vs patients with influenza. JAMA Neurol. 2020. https://doi.org/10.1001/jamaneurol .2020.2730.

8. Carroll E, Lewis A. Catastrophic intracranial hemorrhage in two critically ill patients with covid-19. Neurocrit Care. 2020. https://doi.org/10.1007/ s12028-020-00993-5.

9. Kothari RU, Brott T, Broderick JP, Barsan WG, Sauerbeck LR, Zuccarello M, et al. The ABCs of measuring intracerebral hemorrhage volumes. Stroke. 1996;27:1304-5.

10. Hemphill JC 3rd, Bonovich DC, Besmertis L, Manley GT, Johnston SC. The ICH score: A simple, reliable grading scale for intracerebral hemorrhage. Stroke. 2001;32:891-7.

11. Connors JM, Levy JH. Covid-19 and its implications for thrombosis and anticoagulation. Blood. 2020;135:2033.

12. Goeijenbier M, van Wissen $M$, van de Weg C, Jong E, Gerdes VE, Meijers JC, et al. Review: viral infections and mechanisms of thrombosis and bleeding. J Med Virol. 2012;84:1680-96.

13. Panigada M, Bottino N, Tagliabue P, Grasselli G, Novembrino C, Chantarangkul $\mathrm{V}$, et al. Hypercoagulability of COVID-19 patients in intensive care unit: a report of thromboelastography findings and other parameters of hemostasis. J Thromb Haemost. 2020;18:1738-42.

14. Klok FA, Kruip M, van der Meer NJM, Arbous MS, Gommers D, Kant KM, et al. Incidence of thrombotic complications in critically ill ICU patients with COVID-19. Thromb Res. 2020;191:1248.

15. Moores LK, Tritschler T, Brosnahan S, Carrier M, Collen JF, Doerschug $\mathrm{K}$, et al. Prevention, diagnosis, and treatment of VTE in patients with covid-19: Chest guideline and expert panel report. Chest. 2020. https:// doi.org/10.1016/j.chest.2020.05.559.

16. American Society of Hematology. COVID-19 and VTE/anticoagulation: frequently asked questions. 2020. https://www.hematology.org/covid -19/covid-19-and-vte-anticoagulation. Accessed 6 July 62020.

17. Zhou L, Zhang M, Wang J, Gao J. SARS-CoV-2: Underestimated damage to nervous system. Travel Med Infect Dis. 2020. https://doi.org/10.1016/j. tmaid.2020.101642.

18. Vonck K, Garrez I, De Herdt V, Hemelsoet D, Laureys G, Raedt R, et al. Neurological manifestations and neuro-invasive mechanisms of the severe acute respiratory syndrome coronavirus type 2. Eur J Neurol. 2020;27:1578.

19. Wu Y, Xu X, Chen Z, Duan J, Hashimoto K, Yang L, et al. Nervous system involvement after infection with covid-19 and other coronaviruses. Brain Behav Immun. 2020;87:18

20. Ackermann M, Verleden SE, Kuehnel M, Haverich A, Welte T, Laenger F, et al. Pulmonary vascular endothelialitis, thrombosis, and angiogenesis in covid-19. N Engl J Med. 2020;383:120.

21. van de Veerdonk FL, Netea MG, van Deuren M, van der Meer JW, de Mast Q, Bruggemann RJ, et al. Kallikrein-kinin blockade in patients with covid19 to prevent acute respiratory distress syndrome. Elife. 2020. https://doi. org/10.7554/eLife.57555.

22. Seiffge DJ, Werring DJ, Paciaroni M, Dawson J, Warach S, Milling TJ, et al. Timing of anticoagulation after recent ischaemic stroke in patients with atrial fibrillation. Lancet Neurol. 2019:18:117-26.

23. Paciaroni M, Agnelli G, Falocci N, Caso V, Becattini C, Marcheselli S, et al. Early recurrence and cerebral bleeding in patients with acute ischemic stroke and atrial fibrillation: effect of anticoagulation and its timing: The raf study. Stroke. 2015:46:2175-82.

24. Altavilla R, Caso V, Bandini F, Agnelli G, Tsivgoulis G, Yaghi S, et al. Anticoagulation after stroke in patients with atrial fibrillation. Stroke. 2019;50:2093-100.

25. Divani AA, Majidi S, Luo X, Souslian FG, Zhang J, Abosch A, et al. The abcs of accurate volumetric measurement of cerebral hematoma. Stroke. 2011:42:1569-74.

26. Salazar P, Di Napoli M, Jafari M, Jafarli A, Ziai W, Petersen A, et al. Exploration of multiparameter hematoma $3 \mathrm{~d}$ image analysis for predicting outcome after intracerebral hemorrhage. Neurocrit Care. 2020;32:539-49. 